\title{
Direct measurement of critical Casimir forces
}

\author{
C. Hertlein ${ }^{1}$, L. Helden ${ }^{1}$, A. Gambassi ${ }^{2,3}$, S. Dietrich ${ }^{2,3}$ \& C. Bechinger ${ }^{1}$
}

When fluctuating fields are confined between two surfaces, long range forces arise. A famous example is the quantum electrodynamical Casimir force that results from zero point vacuum fluctuations confined between two conducting metal plates $^{1}$. A thermodynamic analogue is the critical Casimir force: it acts between surfaces immersed in a binary liquid mixture close to its critical point and arises from the confinement of concentra tion fluctuations within the thin film of fluid separating the sur faces ${ }^{2}$. So far, all experimental evidence for the existence of this effect has been indirect ${ }^{35}$. Here we report the direct measurement of critical Casimir force between a single colloidal sphere and a flat silica surface immersed in a mixture of water and 2,6 lutidine near its critical point. We use total internal reflection microscopy to determine in situ the forces between the sphere and the surface, with femtonewton resolution 6 . Depending on whether the adsorp tion preferences of the sphere and the surface for water and 2,6 lutidine are identical or opposite, we measure attractive and repulsive forces, respectively, that agree quantitatively with theoretical predictions and exhibit exquisite dependence on the temperature of the system. We expect that these features of critical Casimir forces may result in novel uses of colloids as model systems.

The simple act of confining a fluid can give rise to new phenomena not observed in the bulk. An intriguing example is the critical Casimir force predicted to occur in binary fluid mixtures close to their critical point; like other critical phenomena, it is characterized by universal scaling functions that depend only on the internal symmetries of the system rather than on its specific material properties ${ }^{7}$. Colloidal particles suspended in binary liquids offer a particularly interesting setting for the experimental observations of such forces. At suffi ciently small particle distances, concentration fluctuations of the solvent become confined between neighbouring colloidal surfaces and modify the pair interaction ${ }^{8}$. If the Casimir interaction strength is comparable to the thermal energy, drastic changes in the phase behaviour are expected. Reversible flocculation of silica colloids in water 2,6 lutidine mixtures close to the critical point has in fact been observed ${ }^{9}$, and critical Casimir forces may be invoked to explain this phenomenon. However, flocculation was observed even far away from the critical point where critical fluctuations are negligible, so it cannot serve as conclusive evidence for the presence of Casimir forces $^{10,11}$.

Our experiments, aimed at directly measuring critical Casimir forces, use a single colloidal sphere and a planar surface immersed in a binary liquid mixture of water and 2,6 lutidine. Forces are deter mined using total internal reflection microscopy (TIRM, see Methods $)^{6}$ which allows in situ measurements with femtonewton resolution $^{12}$ (Fig. 1). The binary liquid mixture has a lower critical demixing point at $T_{\mathrm{C}} \cong 307 \mathrm{~K}$ at a lutidine mass fraction of $c_{\mathrm{L}}^{\mathrm{C}}=0.286$. At temperatures far below the temperatures correspond ing to the demixing line, the binary mixture can be considered as an effectively homogeneous solvent and critical Casimir forces are absent. Under those conditions, the potential of the negatively charged colloidal particle at height $z$ above the surface is given by electrostatic, gravitational and optical forces ${ }^{6}$

$$
\Phi(z)=A \exp (-\kappa z)+G_{\text {eff }} z
$$

where the amplitude of the electrostatic interaction $A$ depends on the surface charges of the particle and the wall ${ }^{6},{ }^{1}{ }^{1}$ is the Debye screen ing length of the solvent, and $G_{\text {eff }}$ is the effective weight of the colloid due to gravity and light pressure from the optical tweezers. For all heights $z$ sampled in our experiment, we see no evidence for disper sion forces. This is due to the rather small differences between the refractive indices $n$ of the water lutidine mixture (1.384) and the silica substrate (1.464).

Because critical Casimir forces depend strongly on the adsorption properties of the confining surfaces ${ }^{7}$, we used different combinations of particles and surfaces that preferentially adsorb either lutidine ('plus' boundary condition) or water ('minus' boundary condition). We used polystyrene particles ${ }^{13}$ of diameter $2 \mathrm{R} \quad 3.69 \mu \mathrm{m}$ with a clear preference for lutidine, and highly charged polystyrene spheres of diameter $2.4 \mu \mathrm{m}$ preferring water. Variation of the adsorption pro perties of the silica substrate is achieved by chemical treatment of its surface with either hexamethyldisilaxane (HMDS) or $\mathrm{NaOH}$, result ing in a preferential adsorption for lutidine and water, respectively.
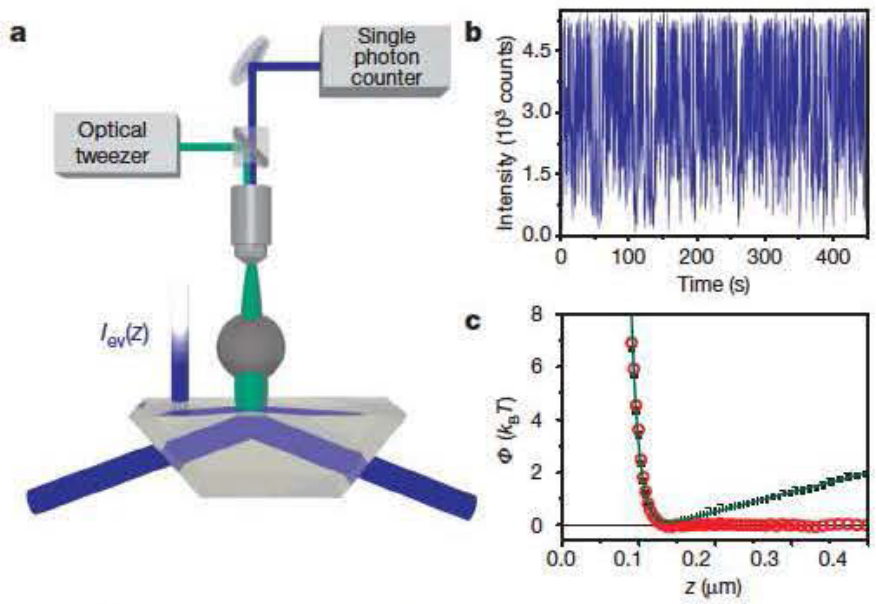

Figure 1| Data acquisition and analysis. a, Scheme of TIRM set up. A colloidal particle undergoing thermal motion within an evanescent field of intensity $I_{\text {ev }}(z)$ scatters light, whose intensity $I_{s c}(z)$ is detected by a single photon counter. Lateral particle diffusion is suppressed by a vertically incident optical tweezer $(\lambda=532 \mathrm{~nm}, P \cong 2 \mathrm{~mW})$. b, $I_{\text {sc }}$ versus time, reflecting particle motion normal to the surface. $c$, Measured interaction potential for a $2.4 \mu \mathrm{m}$ polystyrene particle above a $\mathrm{NaOH}$ treated silica surface (( ) boundary conditions) in a water lutidine mixture at critical composition (black data points). The data points are well fitted by equation (1) (green line). The same data but with the linear contribution $G_{\text {eff }} Z$ subtracted and shifted in vertical direction is also plotted (red data points).

2. Physikalisches Institut, Universitat Stuttgart, Pfaffenwaldring 57, 70569 Stuttgart, Germany. ${ }^{2}$ Max Planck Institut fur Metallforschung, Heisenbergstrasse 3, 70569 Stuttgart, Germany. ${ }^{3}$ Institut fur Theoretische und Angewandte Physik, Pfaffenwaldring 57, Universitat Stuttgart, 70569 Stuttgart, Germany. 
Figure $1 \mathrm{c}$ gives the interaction potential measured for a $2.4 \mu \mathrm{m}$ particle above a $\mathrm{NaOH}$ treated surface (( ) boundary conditions) in a water lutidine mixture at critical composition and $0.3 \mathrm{~K}$ below $T_{\mathrm{C}}$. $T_{\mathrm{C}}$ is determined as the temperature at which a characteristic change in the scattering intensity occurs upon slowly heating the system across the phase transition. The figure shows that our mea sured potential is accurately described by equation (1), with the fit yielding a value of $\kappa^{1} \approx 12 \mathrm{~nm} \pm 3 \mathrm{~nm}$ (s.d.) for the Debye screening length that is in agreement with estimates based on the solvation constant of the mixture ${ }^{14}$. We note that the linear contribution to the interaction potentials (see equation (1)) is identical over the entire temperature range we investigated. For convenience, we have subtracted this contribution from all potentials shown in the remain der of the paper.

Figure 2a shows the particle wall interaction potentials upon approaching $T_{\mathrm{C}}$ from below. Within the investigated temperature range, critical fluctuations of the fluid mixtures contribute only neg ligibly to background scattering and thus do not interfere with the measurements of interaction potentials. The data show that although the temperature varies by only $0.18 \mathrm{~K}$, the approach to $T_{\mathrm{C}}$ results in large changes to the measured potentials as a strong attractive force between particle and surface develops. In combination with electro static repulsion, the attractive force yields a potential well with a depth of almost $10 k_{\mathrm{B}} T$. At the highest temperature, corresponding to $\Delta T \quad T_{\mathrm{C}} \quad T \quad 0.12 \mathrm{~K}$, this well is sufficiently deep that the par ticle hardly escapes from it during the entire measurement. The strong temperature dependence of the potentials close to the critical point is a clear indication of the involvement of critical Casimir forces. The maximum attractive force acting on the particle is esti mated to about $600 \mathrm{fN}$. Considering the linear dependence of such
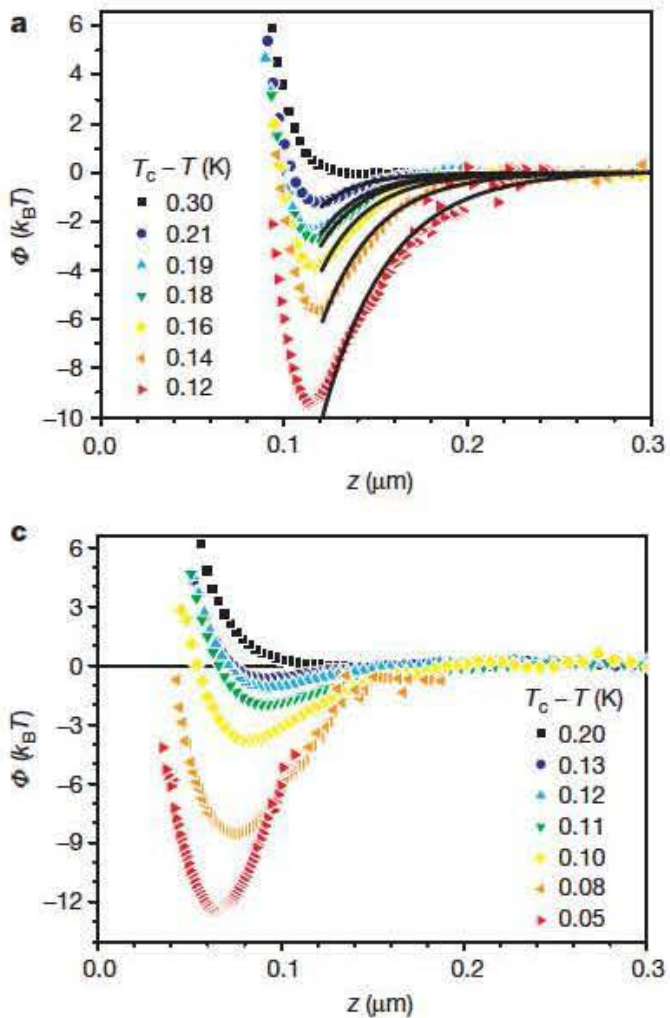

Figure 2 | Critical Casimir potentials between a wall and a particle in a critical water-lutidine mixture. a, Symmetric boundary conditions $(--)$ : $2.4 \mu \mathrm{m}$ hydrophilic particle and $\mathrm{NaOH}$ treated hydrophilic wall. b, Asymmetric boundary conditions $(+-): 3.69 \mu \mathrm{m}$ polystyrene particle preferring lutidine and $\mathrm{NaOH}$ treated hydrophilic wall, c, Symmetric boundary conditions $(++): 3.69 \mu \mathrm{m}$ polystyrene particle and HMDS treated wall. d, Calculated scaling functions for symmetric and asymmetric forces on the particle size, this value is comparable to reported quantum electrodynamical Casimir forces between a metallized col loidal sphere and a conducting flat surface ${ }^{15}$.

Indirect experimental evidence has documented not only attrac tive but also repulsive critical Casimir forces in thin films of classical fluids $s^{5,16}$, with the observations of one study ${ }^{5}$ found to agree with subsequent theoretical predictions ${ }^{17}$. To observe repulsive critical Casimir forces in our system directly, the two surfaces confining the binary fluid mixture must preferentially adsorb different species of the mixture ${ }^{7}$; that is, the boundary conditions for the particles and surface need to be 'plus minus' or 'minus plus': $(+)$ or $(+)$. In an experimental realization of $(+)$ conditions we used $3.69 \mu \mathrm{m}$ polystyrene particles and $\mathrm{NaOH}$ treated substrate, with the inter action potentials measured for this system shown in Fig. 2b. As in the earlier experiment (Fig. 2a), the interaction potential measured at temperatures far below $T_{\mathrm{C}}$ consists only of electrostatic contribu tions. But in contrast to the behaviour seen in Fig. 2a, the repulsive part of the potential curves shifts towards larger $z$ as the temperature approaches $T_{\mathrm{C}}$. This is in qualitative agreement with theoretical predictions ${ }^{7}$ for repulsive critical Casimir forces in the case of asym metric boundary conditions. Treatment of the substrate with HMDS restores symmetric boundary conditions with the $3.69 \mu \mathrm{m}$ polysty rene particles, and the critical Casimir forces measured in this $(++)$ system are indeed attractive (see Fig. 2c).

To extract quantitative information about the critical Casimir potential $\Phi_{\mathrm{C}}(z, T)$, we focus on experimental data obtained at dis tances $z$ that are sufficiently large to render electrostatic interactions negligible. This avoids possible complications due to electrostatic effects, given that it is not a priori clear whether critical fluctuations might affect the counterion distribution. Within the Derjaguin
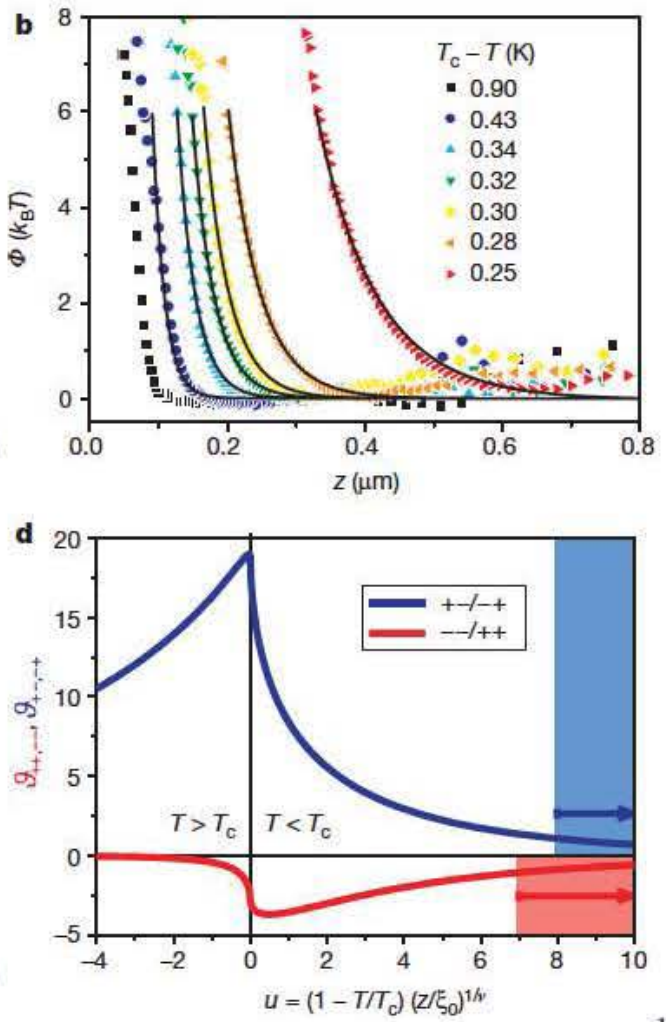

boundary conditions as functions of $u=\left(\begin{array}{ll}1 & \frac{T}{T_{C}}\end{array}\right)\left(\frac{z}{\zeta_{0}}\right)^{1 / v}$ with $u=x^{1 / v}$ for $T<T_{\mathrm{C}}$. The coloured regions indicate the experimentally sampled range. $T$ he solid lines in $\mathbf{a}$ and $\mathbf{b}$ correspond to theoretical calculations with $\xi$ determined as described in the main text. To achieve the best agreement with the theory, the experimental data have been horizontally shifted by the same amount within each panel and within the experimental resolution of $\pm 30 \mathrm{~nm}$. 
approximation, valid for $z \leqq R, \Phi_{\mathrm{C}}$ is expected to scale at the critical concentration as $\frac{\phi_{c}}{k_{a} T}=\frac{R}{z} \vartheta(\xi)$ where $\vartheta$ is a universal scaling function that depends on the boundary conditions. $\xi$ is the bulk correlation length defined as $\xi=\xi_{0}\left(1-\frac{T}{T c}\right)^{-r}$, with the amplitude $\xi_{0}$ reflecting the typical length scale set by the intermolecular pair potential. $v \quad 0.63$ is the universal critical exponent of the three dimensional Ising universality class, relevant for classical binary mixtures. Within the Derjaguin approximation, the scaling function 9 can be calcu lated by expressing it in terms of the scaling function $\vartheta_{\|}(L / \xi)=\frac{F_{\mathrm{C} \|}}{k_{\mathrm{B}} T S} L^{3}$ of the critical Casimir force $F_{C, \|}$ acting between parallel plates with an area $S$ and separated by a distance $L$. This yields $\vartheta(x)=2 \pi \int_{1}^{\infty} \mathrm{d} y\left(y^{-2}-y^{-3}\right) \vartheta_{\|}(x y)$, where $x \quad z / \xi . \vartheta_{\|}$has been deter

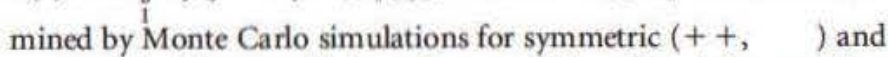
asymmetric $(+,+)$ boundary conditions ${ }^{17}$ and the resulting theoretical prediction for $\mathscr{Y}(x)$ is presented in Fig. $2 \mathrm{~d}$.

The observation that $\mathscr{Y ( x )}$, and thus critical Casimir forces, can change sign depending on the boundary conditions of the system can be understood by considering that the preferences of the confining surfaces for one of the two species present in the fluid mixture impose additional constraints on concentration fluctuations. In the case of symmetric $(++)$ or $(\quad)$ boundary conditions, the fluctuation spectrum is further reduced by the confinement than in a system exhibiting no preferential adsorption; the reduction gives rise to strongly negative $\mathscr{Y}(x)$ values: that is, strong attraction. In the case of asymmetric $(+)$ and $(+)$ boundary conditions, fluctuations in the concentration of opposing species originate at the two confin ing surfaces. At small particle wall distances, these fluctuations inter fere with the tendency of the system to generate concentration fluctuations on the length scale set by the correlation length of the fluid mixture. Confinement in systems with asymmetric boundary conditions thus renders the latter fluctuations energetically less favourable, and so repulsive forces occur.

Theoretical predictions for the interaction potentials arising from critical Casimir forces are shown as solid lines in Fig. $2 \mathrm{a}$ and $\mathrm{b}$, and agree remarkably well with the experimental data. (The data in Fig. $2 \mathrm{c}$ cannot be compared reliably with theory because the $z$ range in which electrostatic contributions are negligible is too narrow.) The

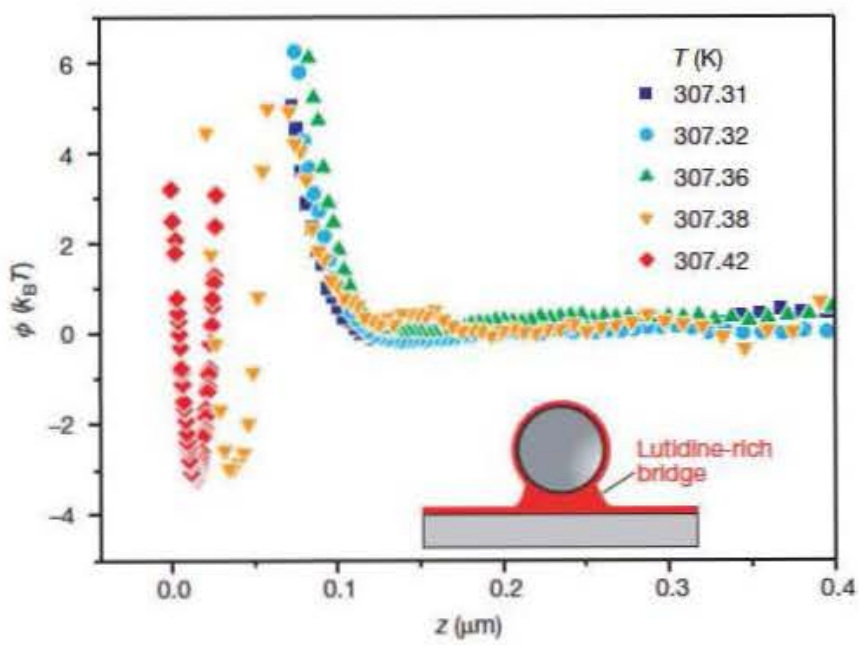

Figure 3 | Off-critical composition. Interaction potentials of a $3.69 \mu \mathrm{m}$ polystyrene particle and a HMDS treated silica wall $((++)$ boundary conditions) at lutidine mass fraction $c_{\mathrm{L}}=0.2$ and temperatures close to the demixing line. The potentials exhibit an abrupt change such that a narrow minimum close to the wall develops, which is interpreted as the formation of a liquid bridge, rich in the preferred component of the mixture, between the partide and the wall, as indicated in the inset. predictions use values for $\xi(T)$ which are optimized to yield the best fits to the experimental data, and which are found to range from 20 to $100 \mathrm{~nm}$. Comparing these $\xi(T)$ values with the theoretically expected algebraic behaviour $\xi=\xi_{0}\left(1-\frac{T}{T_{C}}\right)^{-0.63}$ and treating $\xi_{0}$ and $T_{C}$ as fitting parameters, we have obtained $\xi_{0} 0.18 \mathrm{~nm} \pm 0.02 \mathrm{~nm}$ (s.e.m.) from both experiments in Fig. $2 \mathrm{a}$ and $\mathrm{b}$. This value is con sistent with $\xi_{0} \quad 0.2 \mathrm{~nm} \pm 0.02 \mathrm{~nm}$ (s.e.m.), determined from light scattering experiments ${ }^{18}$. The $T_{C}$ values obtained from the fit are $55 \pm 10 \mathrm{mK}$ (s.e.m.) and $234 \pm 5 \mathrm{mK}$ (s.e.m.), lower than the values experimentally determined in the corresponding experiments sum marized in Fig. $2 a$ and $b$, respectively. We attribute these differences to the difficulties in inferring the absolute value of $T_{\mathrm{C}}$ from the scattering intensity.

We also extended our measurements to binary mixtures that are far from their critical composition, where critical Casimir forces become negligible ${ }^{11}$. For small deviations from the critical lutidine mass fraction $c_{\mathrm{L}}^{\mathrm{C}}$, the temperature dependence of the forces between particles and surfaces is predicted to be almost identical to the tem perature dependence documented in Fig. 2 (ref. 11). This is in agree ment with our observation that measurements for lutidine mass fractions in the range $0.26<q_{2}<0.32$ and using $(++)$ boundary conditions yield potential curves similar to those shown in Fig. $2 c$ (data not shown). During these experiments, we observe no evidence for wetting phenomena, which are strongly suppressed by the curv ature of the spherical particle ${ }^{19}$. However, measurements for $c_{\mathrm{L}} \lesssim 0.2$ result in markedly different potentials.

Figure 3 shows the particle wall interaction potentials measured at several temperatures below but near the demixing line, for a system with $(++)$ boundary conditions and $c_{1} \quad 0.2$. Between 307.31 and $307.36 \mathrm{~K}$, the potentials change very little and are well described by equation (1). But a further increase in temperature by $20 \mathrm{mK}$ results in a sudden shift in the interaction potentials, with a narrow and steep potential well developing dose to the surface. Further slight increases in temperature localize the particle even doser to the surface. Such an abrupt change in the particle wall interaction potential strongly con trasts with the gradual increase of critical Casimir forces over a broad temperature range (compare with Fig. 2c, where Casimir forces increase gradually over a $150 \mathrm{mK}$ temperature range). We note that when using a system with $(++)$ boundary conditions, these abrupt potential changes are only observed on the lutidine poor side of the phase diagram. In the case of hydrophilic particles and walls (( ) boundary conditions), we obtain potential curves similar to those in Fig. 3 when $c_{\text {. }} \geq 0.4$. We attribute the instantaneous snapping of the particle towards the wall to the formation of a liquidbridge that spans the particle and the wall and that is formed by one component of the binary mixture. Our observation that bridge formation occurs only on the side of the phase diagram where the mixture is poor in the component that is being preferentially adsorbed by both surfaces is in agreement with theoretical predictions ${ }^{20,21}$, which indicate that under those conditions surface contributions to the free energy become sufficiently important that the system minimizes its interfacial energy by bridge formation (see also the inset in Fig. 3).

Our results demonstrate that intriguing opportunities for influ encing soft matter systems arise when using solvents near their critical point, where critical fluctuations actively contribute to the interactions between suspended objects. The fundamental nature of these fluctuations ensures that in contrast to interactions such as electrostatic forces, critical Casimir forces exhibit a striking temper ature dependence that offers control over the phase behaviour of suspended objects through minute changes in temperature. This feature may lead to new uses of colloids as model systems. And con sidering that repulsive critical Casimir forces can be generated for any material by suitable surface treatments, we envisage that critical Casimir forces might also find use in micro electromechanical systems where stiction (static friction) due to attractive quantum electrodynamical Casimir forces causes device failure ${ }^{22}$. 


\section{METHODS SUMMARY}

Particle wall potentials were measured with TIRM, where a p polarized laser beam ( $\lambda 473 \mathrm{~nm}, P \approx 2 \mathrm{~mW}$ ) is totally reflected at a glass liquid interface leading to an evanescent field (Fig. 1). The particle's scattered intensity in the evanescent field depends sensitively on its height $z: I_{\mathrm{sc}}(z)=I_{0} \exp (\beta z)$ where $\beta^{-1}$ is the evanescent decay length $(\sim 200 \mathrm{~nm}$ in our experiments $)$ and $I_{0}=I_{\mathrm{sc}}(z=0)($ refs 6,23$)$. From the intensity histogram we obtain the equili brium distance distribution $P(z)$ which yields, via the Boltzmann factor, the particle wall interaction potential $\Phi(z)$ up to a constant ${ }^{6}$. The wall position $\left(\begin{array}{ll}z & 0\end{array}\right)$ is obtained with an accuracy of $\pm 30 \mathrm{~nm}$ from the strong $z$ dependence of the particle's effective vertical diffusion coefficient owing to hydrodynamic interactions ${ }^{24}$. Data were acquired with a frequency of $250 \mathrm{~Hz}$, typically over $15 \mathrm{~min}$.

As sample cell we used a $200 \mu \mathrm{m}$ thick silica cuvette containing the critical mixture and a very small amount of colloidal particles. It is optically matched to the prism and thermally coupled to a flow thermostat, providing a temperature stability of $0.01 \mathrm{~K}$ (Lauda, model RK20). The bottom of the cell was coupled to an indium tin oxide coated glass substrate, acting as the heater. Together with a platinum resistor ( $\mathrm{Pt} 100$ ), the heater was connected to a temperature controller (Eurotherm), providing a temperature stability of $\pm 5 \mathrm{mK}$ over several hours.

Two kinds of beads were used as probe particles: $3.69 \mu \mathrm{m}$ diameter polysty rene particles crosslinked with divinyl benzene (Bangs Laboratories, type FS05F) with preferential adsorption of lutidine, and $2.4 \mu \mathrm{m}$ diameter hydrophilic poly styrene spheres with high surface charge density of $10 \mu \mathrm{Ccm}{ }^{2}$ (IDC, type 1 2400). Sample cells were either exposed to HMDS vapour overnight or rinsed with $0.1 \mathrm{M} \mathrm{NaOH}$ for $30 \mathrm{~min}$, leaving the surface hydrophobic or hydrophilic, respectively. The cell was closed with Teflon plugs allowing for consecutive use for multiple days.

1. Casimir, H. B. G. On the attraction between two perfectly conducting plates. Proc. Koninklijke Nederlandse Akad. Wetenschappen B51, 793795 (1948).

2. Fisher, M. E. \& de Gennes, P. G. Phenomena at the walls in a critical binary mixture. C. R. Acad. Sci. Paris B 287, 207209 (1978).

3. Ganshin, A. Scheidemantel, S., Garcia, R. \& Chan, M. H. W. Critical Casimir force in ${ }^{4}$ He films: confirmation of finite size scaling. Phys. Rev. Lett. 97, 075301 (2006).

4. Garcia, R. \& Chan, M. H. W. Critical Casimir effect near the ${ }^{3} \mathrm{He}{ }^{4} \mathrm{He}$ tricritical point. Phys. Rev. Lett. 88, 086101 (2002).

5. Fukuto, M., Yano, Y. F. \& Pershan, P. S. Critical Casimir effect in three dimensional Ising systems: measurements on binary wetting films. Phys. Rev. Lett. 94, 135702 (2005).

6. Prieve, D. C. Measurement of colloidal forces with TIRM. Adv. Colloid Interf. Sci. 82,93125 (1999).
7. Krech, M. Fluctuation induced forces in critical fluids. J. Phys. Cond. Matt. 11, R391 R412 (1999).

8. Hanke, A., Schlesener, F., Eisenriegler, E. \& Dietrich, S. Critical Casimir forces between spherical particles in fluids. Phys. Rev. Lett. 81, 18851888 (1998).

9. Beysens, D. \& Estéve, D. Adsorption phenomena at the surface of silica spheres in a binary liquid mixture. Phys. Rev. Lett. 54, 21232126 (1985).

10. Beysens, D. \& Narayanan, T. Wetting induced aggregation of colloids. J. Stat. Phys. 95, 9971008 (1999).

11. Schlesener, F., Hanke, A. \& Dietrich, S. Critical Casimir forces in colloidal suspensions. J. Stat. Phys. 110, 9811013 (2003).

12. Rudhardt, D., Bechinger, C. \& Leiderer, P. Repulsive depletion interactions in colloid polymer mixtures. J. Phys. Cond. Matt. 11, 1007310078 (1999).

13. Gallagher, P. D., Kurnaz, M. L. \& Maher, J. V. Aggregation in polystyrene sphere suspensions in near critical binary liquid mixtures. Phys. Rev. A. 46, 77507755 (1992).

14. Gallagher, P. D. \& Maher, J. V. Partitioning of polystyrene latex spheres in immiscible critical liquid mixtures. Phys. Rev. A. 46, 20122021 (1992).

15. Mohideen, U. \& Roy, A. Precision measurement of the Casimir force from 0.1 to 0.9 um. Phys. Rev. Lett. 81, 45494552 (1998).

16. Rafai, S., Bonn, D., \&. Meunier, J. Repulsive and attractive critical Casimir forces. Physica A 386, 3135 (2007).

17. Vasilyev, O., Gambassi, A., Maciolek, A. \& Dietrich, S. Monte Carlo simulation results for critical Casimir forces. Europhys. Lett. 80, 60009 (2007).

18. Gülari, E., Collings, A. F., Schmidt, R. L. \& Pings, C. J. Light scattering and shear viscosity studies of the binary system 2,6 lutidine water in the critical region. J. Chem. Phys. 56, 61696179 (1972).

19. Bieker, T. \& Dietrich, S. Wetting of curved surfaces. Physica A 252, 85137 (1998)

20. Dobbs, H. T. Darbellay, G. A. \& Yeomans, J. M. Capillary condensation between spheres. Europhys. Lett. 18, 439444 (1992).

21. Bauer, C., Bieker, T. \& Dietrich, S. Wetting induced effective interaction potential between spherical particles. Phys. Rev. E 62, 53245338 (2000).

22. Ball, P. Feel the force. Nature 447, 772774 (2007).

23. Helden, L. et al. Single particle evanescent light scattering simulations for total internal reflection microscopy. Appl. Opt. 45, 72997308 (2006).

24. Bevan, M. A. \& Prieve, D. C. Hindered diffusion of colloidal particles very near to a wall: revisited. J. Chem. Phys. 113, 12281236 (2000)

Acknowledgements We thank A. Maciołek for inspiring discussions and F. Schlesener, R. Dullens and D. Marr for comments and C. Mayer for sample preparation. This work is financially supported by the Deutsche Forschungsgemeinschaft. 